\title{
COMPORTAMIENTO DE LA ACTIVIDAD EN LOS MÚSCULOS PRIMARIOS DEL DISPARO, EN DEPORTISTAS DE SELECCIÓN COLOMBIA DE ARCO RECURVO
}

\section{BEHAVIOR OF THE ACTIVITY IN THE PRIMARY MUSCLES INVOLVED IN ARCHERY AT THE TIME OF THE SHOOTING, IN ATHLETES OF THE COLOMBIAN NATIONAL RECURVE BOW TEAM.}

\author{
José Armando Vidarte Claros*, Andrés Felipe Marín Villada** Francia Restrepo de Mejía
}

\begin{abstract}
Resumen
Conocer los cambios que se suceden después del desarrollo de un protocolo de fuerza, permite optimizar la calidad en las actividades físicas más específicas que fundamentan el tiro con arco recurvo, donde el óptimo desarrollo del atleta facilite desde el conocimiento científico sentar las bases teóricas y prácticas para el manejo de protocolos adecuados y acordes a las necesidades del deportista y del contexto deportivo.
\end{abstract}

Objetivo. Determinar el comportamiento neuromuscular de miembros superiores de tres deportistas de la selección Colombia de arco recurvo durante la fase de disparo, a partir de la aplicación de un programa de entrenamiento de la fuerza.

Materiales y métodos. Se realizó una investigación de tipo descriptiva, series de casos a través del cual se realizó la valoración de una serie de sujetos tipo. Fueron tres deportistas seleccionados por Colombia para representarnos como selección nacional en las diferentes competencias de carácter internacional, que están cumpliendo el ciclo olímpico para acceder a un cupo a los próximos juegos olímpicos, Londres 2012.

Conclusiones. Se evidenciaron cambios morfológicos de los deportistas, en relación con los objetivos biológicos y pedagógicos inherentes a la planificación. La fuerza juega un papel decisivo en la ejecución técnica. En muchos casos, el fallo técnico se produce por falta de coordinación o habilidad y por déficit de fuerza en los grupos musculares que intervienen en una fase concreta del movimiento.

Palabras clave: Actividad física, electromiografía, fuerza, ecografía, desarrollo muscular (Descriptores DeCS).

* Ph.D. Ciencias de laActividad Física y el Deporte.Profesor titular en la Universidad Autónoma de Manizales, Departamento de Movimiento Humano, Facultad de Salud. Líder Grupo de investigación Cuerpo - Movimiento. Email: jovida@autonoma.edu.co

** Magíster en Intervención Integral en el deportista.Preparador Físico de la Federación colombiana de tiro con arco recurvo. Email: afelipemarin27@hotmail.com

*** Médico, Fisiatra, Ph.D. Niñez Infancia y Juventud.Profesora Universidad Autónoma de Manizales, Email: francia.restrepo@hotmail.com 


\section{Abstract}

Understanding the changes that occur after the development of a protocol of force enables us to optimize the quality of the specific physical activities underlying the recurve archery, where the optimal development of the athlete facilitates, from a scientific point of view, lay the theoretical and practical basis for handling protocols that are appropriate and consistent with the needs of the athlete and the sporting context. Objective: To determine the neuromuscular behavior of the upper limbs of three athletes of the Colombian national recurve bow team during shooting phase, from the application of a strength training program. Materials and methods: A descriptive research was conducted, using series of cases through which the assessment was made of a series of type subjects. There were three athletes selected by Colombia to represent us as national team in different international competitions, who are taking part in the Olympic cycle in order to gain a place in the next Olympic Games, London 2012. Conclusions: Morphological changes in athletes, in relation to biological and pedagogical objectives inherent in the planning were evidenced. The strength played a decisive role in the technical execution. In many cases, the technical failure is caused by lack of coordination or skill, and strength deficit in the muscle groups involved in a particular phase of the movement.

Key words: Physical Activity, Electromyography, Strength, ultrasound, muscle development (Descriptors in Health Sciences)

\section{Introducción}

El tiro con arco es un deporte de precisión clasificado como deporte aficionado en Colombia (Coldeportes, Ley 181 de 1995) que está constituido por dos modalidades esenciales, condicionadas en dependencia de la implementación utilizada, el arco compuesto y el arco recurvo, de las cuales la última, por tener más dependencia de las cualidades físicas, fisiológicas y mentales del atleta, es clasificada como deporte del ciclo olímpico. Teniendo en cuenta que el tiro con arco recurvo se fundamenta en gran parte por la óptima ejecución de la técnica, las pocas investigaciones y publicaciones son destinadas al desarrollo de la misma. Se reconoce como las capacidades físicas y mentales como la fuerza, la coordinación, la resistencia, la concentración y la competitividad, condicionan de gran forma el rendimiento deportivo (Lee et al., 2005), por lo tanto no se puede omitir o excluir estas capacidades del proceso de formación de los arqueros. En este sentido, al igual que otros deportes tanto individuales como colectivos el tiro con arco requiere no solamente del desarrollo de la técnica deportiva, sino, también el desarrollo y fortalecimiento de las capacidades físicas, entre ellas, el desarrollo de la fuerza general y especial en los miembros superiores.

Es necesario el entrenamiento de la fuerza a partir de un programa riguroso y sistemático que permita conocer el comportamiento neuromuscular de los grupos musculares involucrados en el gesto técnico, aspecto que aún no se ha llevado a cabo, ya que actualmente los procesos desarrollados en esta modalidad deportiva, han sido espontáneos, sin sustento científico. Al realizar el entrenamiento de esta capacidad física, se incide directamente a nivel celular mediante las manifestaciones de la fuerza, (fuerza máxima, rápida y de resistencia), el entrenamiento sistemático favorece la capacidad de rendimiento físico-deportivo, consolida capacidades psicológicas, mejora la postura corporal; aumenta la funcionalidad del sistema neuro muscular, respiratorio, cardiovascular, metabólico y óseo, afectando positivamente la salud, por tanto, la fuerza puede ser estimulada a través del entrenamiento por diferentes métodos, los cuales conducirán a la adaptación biológica del sistema músculo esquelético. Así mismo la medición de la fuerza aplicada es uno de los criterios de mayor validez para hacer una valoración de la propia técnica deportiva. La fuerza aplicada se mide a través de los cambios de aceleración de las resistencias externas y por la deformación que se produce en los dinamómetros, tanto por efecto de la tensión como de la compresión que se ejerce sobre ellos.

La mayoría de estudios desarrollados sobre la capacidad de la fuerza se han establecido sobre deportes como judo, tenis de mesa y lucha (Carratalá et al. 2003; Mansilla et al. 2006; Berdejo et al. 2009), en el deporte del tiro se ha realizado un trabajo que da cuenta del manejo del protocolo de contraste pre- isométrico como método para el incremento de la fuerza específica (Herrera, 2009), en el cual se encontraron mejoras de la fuerza general en un $23 \%$; 
intuyendo que el trabajo de fuerza es un factor fundamental para el desarrollo eficiente del gesto técnico y el incremento de las puntuaciones del arquero. En la actualidad Colombia ha participado como equipo en los juegos olímpicos celebrados en Beijing (China) en 2008, con el equipo recurvo femenino; este aspecto permitió que la federación colombiana de tiro con arco apoyara la conformación del equipo masculino con la intencionalidad de participar en el ciclo olímpico.

El equipo masculino actualmente se encuentra conformado por tres deportistas con poca experiencia en el ámbito internacional y muchas capacidades a desarrollar gracias a su edad joven y con mucho potencial de desarrollo, desde un proceso metódico a través de los programas de entrenamiento deportivo, con el fin de optimizar las capacidades condicionales de la modalidad, creando bases sólidas en la manera adecuada de desarrollar planificaciones específicas del deporte en cuanto a preparación física específica se refiere, con entrenamientos direccionados a las necesidades físicas del deportista mejorando así la calidad de trabajo del trabajo, capacitando los músculos específicos del disparo para una ejecución más fina y limpia, acondicionándolos a la alta competencia y disminuyendo el riesgo de lesión (Torres et al. 2005; Mansilla etal. 2004; Ergen et al. 2004,2008; Lee et al. 2009). Sumado a lo anterior el proceso de intervención desarrollado evidenció cambios en los músculos específicos del disparo después de potenciar la fuerza a través del programa estructurado en busca del desarrollo óptimo de dichos músculos y permitió determinar que el proceso de entrenamiento condiciona a los atletas en la búsqueda de altos logros.

El desarrollo de la fuerza específica se basa en el entrenamiento de los músculos que actúan directamente sobre el movimiento realizado por el atleta en competición, por lo tanto las cargas se imponen en forma de tensión neuromuscular para el desarrollo típico de cada deporte y amarrado al desarrollo de la ejecución técnica de la modalidad (Lee et al. 2005). En el desarrollo de la fuerza especial se evidencia la necesidad del mejoramiento especifico neuromuscular por lo que se hace importante la coordinación la cual se consigue por medio del desarrollo tanto intermuscular como el intramuscular, definiendo el tipo de desarrollo de las tenciones 0 acciones de trabajo en la técnica del deporte (Mansilla et al. 2006; Lee et al. 2009).

Algunos estudios han demostrado que la realización de un programa sistémico de fuerza para el desarrollo de la coordinación intramuscular, es acompañada por un incremento significativo en la producción de la fuerza, siempre y cuando la intensidad y la duración del periodo de entrenamiento sean suficientes y cualquier tipo de entrenamiento tendrá la capacidad de mejorar cualquiera de las variables de la fuerza, ya se máxima, veloz, potencia y en dependencia con la fuerza especifica que se quiere desarrollar se definirá la intensidad el volumen y cadencia del entrenamiento (Lee et al. 2009).

En esta investigación se utilizaron como métodos de evaluación de la fuerza, la electromiografía, la ecografía y la evaluación de la fuerza máxima isométrica. De tal manera que en el presente estudio se pretendió determinar el comportamiento neuromuscular de miembros superiores de tres deportistas de la selección Colombia de arco recurvo durante la fase de disparo, a partir de la aplicación de un programa de entrenamiento de la fuerza.

\section{Materiales y métodos}

La presente investigación se inscribe dentro de los estudios Descriptivos series de casos; a través del cual se realizó la valoración de una serie de sujetos tipo, los cuales a nivel nacional son exclusivos en el tipo de actividad que realizan. La muestra fueron tres deportistas seleccionados por Colombia que están cumpliendo el ciclo olímpico, los cuales cumplieron con los criterios de inclusión que fueron ser deportistas selección Colombia, categoría elite de la modalidad recurvo masculino que hayan aceptado ingresar al estudio por medio del consentimiento informado.

Los deportistas se concentraron en una sede deportiva propuesta por la federación colombiana de tiro con arco, cerca del lugar de las evaluaciones y los controles facilitando el proceso de evaluación y disposición completa-de éstos para la ejecución de los programas de desarrollo de fuerza específica. Fueron utilizadas como técnicas la observación realizada durante el proceso de entrenamiento y en las diferentes evaluaciones que se hicieron-a los deportistas pre y postest. El instrumento de recolección de información fue un formato donde se consignaron todos los valores obtenidos mediante las diferentes pruebas. Se concertó con el médico de la selección Colombia los test de valoración médica, en la clínica Risaralda se realizó la ecografía músculo esquelética en fase de relajación y en tensión muscular ejercida mediante la técnica habitual del disparo. En el laboratorio de movimiento humano de la universidad tecnológica de Pereira se realizaron los test de fuerza en la multifuncional direccionada por un sistema de 
galgas con un software globus ergo sistem, TCS suit para evaluar la fuerza máxima isométrica. En el laboratorio de neurofisiología de la Universidad Autónoma de Manizales se realizó la electromiografía en los músculos que priman el disparo deltoides trapecio medio y pectoral mayor, en fase crítica del anclaje.-El desarrollo del programa de entrenamiento de la fuerza se desarrolló durante cuatro meses.

Las implicaciones éticas del estudio tuvieron en cuenta lo planteado por la resolución 008430 de MinProtección artículo 11, donde se asumió como una investigación de riesgo mínimo. Además se firmaron los consentimientos informados y se contó con la aprobación en acta del comité de bioética de la Universidad Autónoma de Manizales.

\section{Resultados}

En el siguiente apartado se muestran los resultados del presente estudio. Por el tipo de investigación se resalta que se muestran de acuerdo al comportamiento en cada uno de los casos participantes en el estudio.

Deportista 1: El estudio del desempeño de los grupos musculares estimulados durante la intervención reveló diferencias en cada uno de los deportistas evaluado en el sistema software Globus ergo Sistem TC 4400. Medición pre y post test, (tabla 1). El deportista tiene 16 años; el peso en la medi- ción inicial fue de $68 \mathrm{Kg}$ y en la evaluación final de 71,3 $\mathrm{Kg}$. La talla es de $183 \mathrm{~cm}$, el porcentaje graso en la medición inicial de 15,7\% y al finalizar de 16,2\%.

El análisis ecográfico (pre-test y pos-test) se realizó en los tendones infra espinoso, supra espinoso y redondo menor. A nivel tendinoso del manguito rotador derecho (MRD) y manguito rotador izquierdo (MRI), el análisis muestra cómo el deportista tuvo inicialmente una media en MRD de $3.13 \mathrm{~mm}$ en comparación con una media en el post-test, de $4.76 \mathrm{~mm}$. En el MRI (pre-test) tuvo una media de 3-8 $\mathrm{mm}$ y en el post-test de $4.8 \mathrm{~mm}$, con normalidad en su formación tendinosa. En el momento de pretest, se diagnosticó: Patrón fibrilar del bíceps braquial normal, sin presencia de líquido en su vaina. Tendón supra espinoso e infraespinoso de grosor normal, sin signos de ruptura parcial o total; músculos del cuadrilátero con asimetría, con predominio del redondo menor izquierdo; no hay anormalidades paralabrales; no se observan irregularidades del contorno humeral; ecografía del hombro comparativa estática y dinámica dentro de los límites normales. En el pos-test se observan cambios significativos en la trofia muscular y tendinosa, con mayor grosor y elasticidad del tejido; sin presencia de inflamación o deterioro del mismo: Patrón fibrilar del bíceps braquial, normal, sin presencia de líquido en su vaina. Tendones supraespinoso e infraespinoso de grosor normal, sin signos de ruptura parcial o total; músculos

Tabla 1. Comportamiento de la fuerza del deportista 1 durante el pretest y postest de los deportistas participantes en el estudio

\begin{tabular}{|c|c|c|c|c|}
\hline Atleta & Músculo & Pretest & Postest & Diferencia \\
\hline \multirow[t]{5}{*}{ Deportista 1} & Pecho & $40 \mathrm{Kg}$. & $65 \mathrm{Kg}$. & $25 \mathrm{~kg}$. \\
\hline & Tríceps & $30 \mathrm{Kg}$. & $45 \mathrm{Kg}$. & $15 \mathrm{~kg}$. \\
\hline & Hombro & $40 \mathrm{Kg}$. & $60 \mathrm{Kg}$ & $20 \mathrm{~kg}$. \\
\hline & Espalda & $80 \mathrm{Kg}$. & $137 \mathrm{Kg}$. & $57 \mathrm{Kg}$. \\
\hline & Cuádriceps & $70 \mathrm{Kg}$ & $80 \mathrm{Kg}$ & $10 \mathrm{Kg}$. \\
\hline \multirow[t]{5}{*}{ Deportista 2} & Tríceps & $25 \mathrm{Kg}$. & $41 \mathrm{Kg}$. & $16 \mathrm{Kg}$. \\
\hline & Hombro & $35 \mathrm{Kg}$. & $43 \mathrm{Kg}$. & $8 \mathrm{Kg}$. \\
\hline & Espalda & $65 \mathrm{Kg}$. & $93 \mathrm{Kg}$. & $28 \mathrm{Kg}$. \\
\hline & Cuádriceps & $60 \mathrm{Kg}$. & $75 \mathrm{Kg}$. & $15 \mathrm{Kg}$. \\
\hline & Pecho & $36 \mathrm{Kg}$. & $50 \mathrm{Kg}$ & $14 \mathrm{Kg}$. \\
\hline \multirow[t]{5}{*}{ Deportista 3} & Pecho & $30 \mathrm{Kg}$. & $35 \mathrm{Kg}$. & $5 \mathrm{Kg}$. \\
\hline & Tríceps & $20 \mathrm{Kg}$. & $29 \mathrm{Kg}$. & $9 \mathrm{Kg}$. \\
\hline & Hombro & $37 \mathrm{Kg}$. & $43 \mathrm{Kg}$. & $6 \mathrm{Kg}$. \\
\hline & Espalda & $50 \mathrm{Kg}$. & $75 \mathrm{Kg}$. & $25 \mathrm{Kg}$. \\
\hline & Cuádriceps & $69 \mathrm{Kg}$. & $80 \mathrm{Kg}$. & $11 \mathrm{Kg}$. \\
\hline
\end{tabular}


del cuadrilátero con asimetría, con predominio del redondo menor izquierdo; no hay anormalidades paralabrales; no se observan irregularidades del contorno humeral; ecografía del hombro comparativa estática y dinámica, dentro de los límites normales.

En los resultados de la electromiografía el deportista presenta una disminución de la fatiga muscular en el deltoides derecho de $117 \mathrm{~Hz}$, mientras que en el deltoides izquierdo fue de $7.8 \mathrm{~Hz}$. En el trapecio derecho no hubo cambios, mientras que en el izquierdo, la disminución fue de $7.8 \mathrm{Htz}$. Al revisar la muestra integrada de la electromiografia IEMG, relacionada con los datos de fuerza isométrica de contracción intramuscular, se encontró como en el deltoides derecho hubo una reducción de la necesidad de fuerza isometrica a nivel intramuscular, durante el postest (11.2 $\mu$ v.seg), comparado con la del deltoides izquierdo, que fue de 3.1uv.seg. En el trapecio derecho hubo decrecimiento de la contracción isometrica intramuscular en el postest (3.12 $\mu$ v.seg ), mientras que en el trapecio derecho, se presentó un aumento de la contracción muscular en el postest (5.22 $\mu \mathrm{v}$.seg ). El deportista presentó los mayores cambios pre y post test -tanto de la fuerza referenciada por el IEMG como la fatiga referenciada por la MDF- en el deltoides derecho (figura 1). En la descripción del potencial de acción de las unidades motoras reclutadas para el esfuerzo (PUM), se encontró en los deltoides un decrecimiento de la amplitud del esfuerzo realizado para la contracción de 202.02 y $212.12 \mu$ v.seg respectivamente. Los cambios en el trapecio derecho incluyen la disminución de la necesidad de esfuerzo en 6.73 $\mu v . s e g$, mientras, en el trapecio izquierdo, hubo un aumento de $393.06 \mu \mathrm{v}$.seg. Los resultados indican que el reclutamiento de unidades motoras para el esfuerzo en ambos deltoides y en el trapecio derecho fue menor, mientras que en el trapecio izquierdo, el potencial de reclutamiento de unidades motoras tuvo un mayor aumento.

Deportista 2: Tiene 22 años; el peso en la medición inicial fue de $58 \mathrm{Kg}$ y $62 \mathrm{Kg}$ en la medición final (tabla 1). La talla de $169 \mathrm{cms}$, el porcentaje graso en la medición inicial de $11,1 \%$ y $10,3 \%$ al finalizar. El diagnóstico de la ecografía muestra patrón fibrilar del bíceps braquial normal, sin presencia de líquido en su vaina, tendones supraespinoso e infraespinoso de grosor normal sin signos de ruptura; los músculos del cuadrilátero con asimetría, con predominio del redondo menor izquierdo; no hay anormalidades paralabrales; no se observan irregularidades del contorno humeral; la ecografía del hombro comparativa estática y dinámica dentro de los límites normales. En el postest se encontró cambios en la trofia muscular y tendinosa con mayor grosor y elasticidad del tejido, sin presencia de inflamación o deterioro del mismo; patrón fibrilar del bíceps braquial normal, sin presencia de líquido en su vaina. Tendón supraespinoso e infraespinoso de grosor normal, sin signos de ruptura; músculos del cuadrilátero con asimetría, con predominio del redondo menor izquierdo; no hay anormalidades paralabrales; no se observan irregularidades del contorno humeral; ecografía del hombro comparativa estática y dinámica dentro de los límites normales; en el análisis conjunto de los resultados del estado tendinoso del manguito rotador se evidenció que: En el deportista hubo cambios positivos a nivel de trofia tendinosa, con una media en el pre-test en MRD de 5,76 mm y en el posttest, de 7,33 mm. En MRI, un pre-test de 3,83 $\mathrm{mm}$ y un post-test de $3.90 \mathrm{~mm}$, con normalidad en su formación tendinosa, en relación a la electromiografía, se pudo evidenciar una disminución de la fatiga a nivel de los cuatro músculos evaluados. Se describe un mayor cambio en el músculo deltoides derecho, con un decrecimiento de $93,96 \mathrm{~Hz}$. El músculo que menos cambios presentó fue el deltoides izquierdo, con un decrecimiento de $15,6 \mathrm{~Hz}$ a la frecuencia o fatiga muscular.

Respecto a la fuerza de contracción evaluada en IEMG, los cambios que se describen muestran un decrecimiento en la necesidad de generar la fuerza de contracción isométrica a un mismo estímulo en los músculos primarios evaluados -en la ejecución del gesto técnico-. Los cambios más notables se presentan en el trapecio derecho, con un decrecimiento de $21.6 \mu \mathrm{v}$.seg y en el deltoides derecho, que presentó el menor de todos: $4.6 \mu v$.seg. Los mayores cambios pre-post de la fuerza referenciada por el IEMG se presentaron en el deltoides izquierdo. La fatiga referenciada por la MDF en el deltoides derecho, datos referenciados en el cuadro como frecuencia media y mediana a través del tiempo (figura 2). En la descripción del potencial de acción de las unidades motoras reclutadas para el esfuerzo (PUM), se encontró en los deltoides un decrecimiento de la amplitud del esfuerzo realizado para la contracción de $224.43 \mu$ v.seg para el derecho y $274.02 \mu v$.seg para el izquierdo.

Deportista 3: El nivel de cumplimiento del plan de entrenamiento de la fuerza fue del $54 \%$, con un total de 853 repeticiones (tabla 1). En la ecografía se encontró en el pretest un leve abombamiento e irregularidad de los tendones componentes del manguito izquierdo con las maniobras de tensión muscular; patrón fibrilar del bíceps braquial normal, sin presencia de líquido en su vaina; ten- 

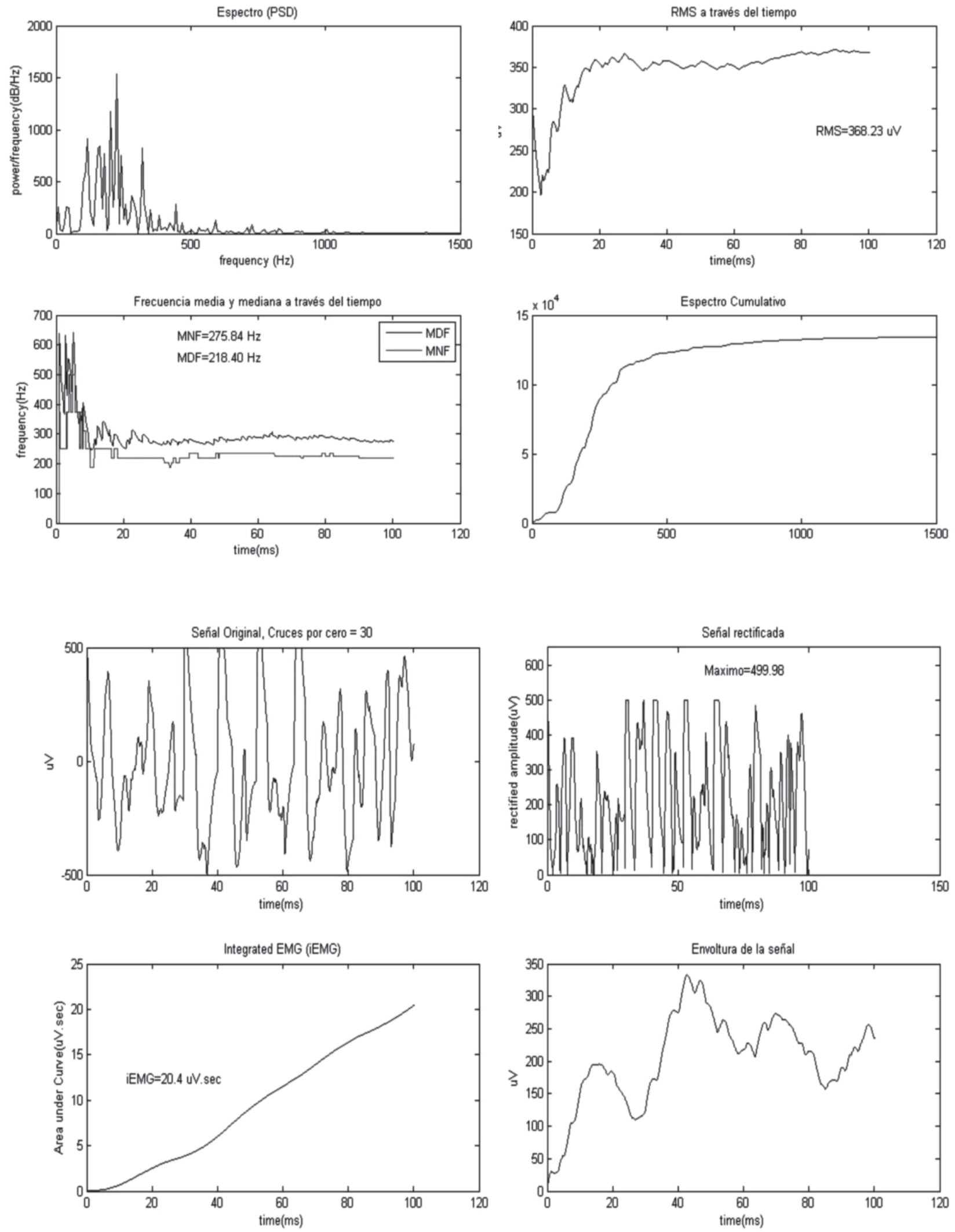

Figura 1. EMG Pretest- Postest deltoides derecho deportista 1 

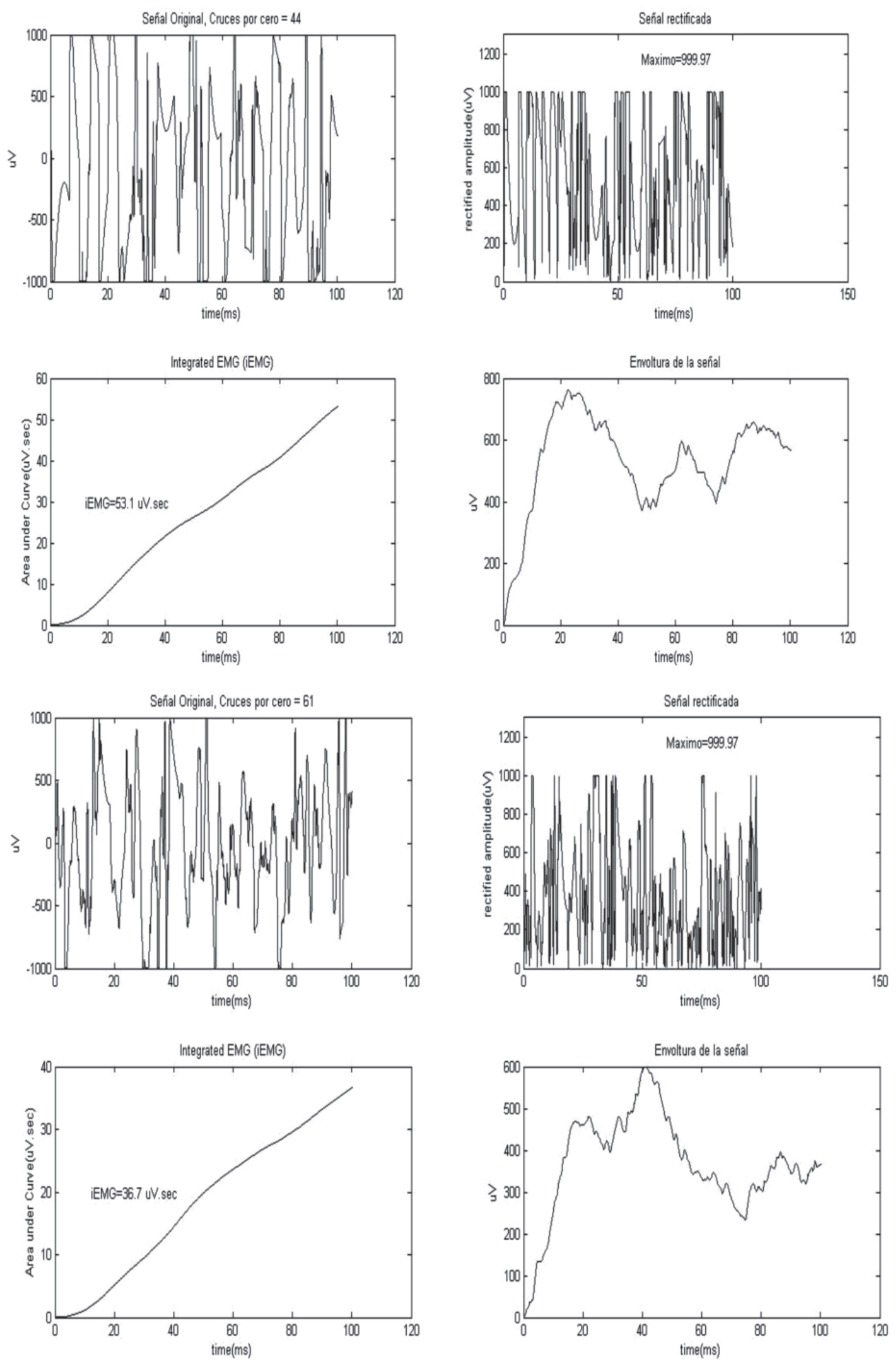

Figura 2. EMG Pretest - Postest deltoides izquierdo, deportista 2 
dón supraespinoso e infraespinoso de grosor normal, sin signos de ruptura; músculos del cuadrilátero con asimetría, con predominio del redondo menor izquierdo; no hay anormalidades paralabrales; no se observan irregularidades del contorno humeral; ecografía del hombro comparativa estática y dinámica dentro de los límites normales. En el postest se encontró abombamiento y pinzamiento del manguito rotador izquierdo, a la tensión muscular, se observan las inserciones del manguito rotador con abombamiento y rose contra el borde inferior del acromion. Se evidenciaron los siguientes cambios a nivel del manguito de los rotadores: en el MRD, el pre-test arrojo una media de $3,30 \mathrm{~mm}$ y el post test de $5,26 \mathrm{~mm}$, con un abombamiento anormal en el supra espinoso derecho. En MRI presentó un pre-test de 3, $80 \mathrm{~mm}$ y un post-test de 3, $56 \mathrm{~mm}$.

Con relación a la electromiografía, el estudio de la media de frecuencia, evidencia un decrecimiento en la fatiga de deltoides de $23,04 \mathrm{~Hz}$ y $15,6 \mathrm{~Hz}$ en el deltoides derecho y trapecio izquierdo, un aumento de la fatiga de $15,9 \mathrm{~Hz}$ y $70,2 \mathrm{~Hz}$ respectivamente. La integrada de electromiografia IEMG, relacionada con la fuerza isometrica de contracción intramuscular muestra un decrecimiento en el deltoides derecho de la necesidad de fuerza isometrica a nivel intramuscular en el postest 1,96 $\mu \mathrm{v}$.seg, comparado con el deltoides izquierdo $6 \mu \mathrm{v}$.seg. Se decrecimiento en el trapecio derecho en el postest $4,2-\mu v$.seg, mientras que en el trapecio derecho hubo un aumento de la contracción muscular en el postest $8,9 \mu \mathrm{v}$. seg.

En la descripción del potencial de acción de las unidades motoras reclutadas para el esfuerzo (PUM), se encontró un decrecimiento de la amplitud del esfuerzo realizado para la contracción de 14,14 $\mu \mathrm{v}$.seg en el deltoides derecho y de $114,56 \mu \mathrm{v}$.seg en el deltoides izquierdo, el trapecio aumentó la amplitud del esfuerzo para la contracción $169,19 \mu \mathrm{v}$.seg y $31,47 \mu \mathrm{v} . \mathrm{seg}$. El reclutamiento de unidades motoras para el esfuerzo en ambos deltoides fue menor, mientras que en los trapecios derecho e izquierdo, el potencial de reclutamiento de unidades motoras tuvo un aumento en la comparación del pre y el postest (figura 3).

\section{Discusión}

Como consecuencia de adaptaciones funcionales y biológicas, los músculos implicados reclutaron menos unidades motoras durante un mismo estímulo aplicado en dos momentos diferentes de la preparación, produciéndose una activación de las fibras de contracción lenta reclutadas en el programa de entrenamiento y en menor cantidad de las fibras de contracción rápida de tipo I y II, datos arrojados en diferentes estudios (González et al. 2011; Keijo et al. 2007; Álvarez, et al., 2006), que al ser comparados con el presente estudio permiten establecer como la señal de amplitud de la onda y las medias de las frecuencias disminuyeron en el postest, en la mayoría de los casos.

Estudios sobre el comportamiento de la fuerza máxima después de diez semanas de entrenamiento progresivo de la fuerza señalan que el incremento significativo de la fuerza está acompañado por un incremento de la actividad neural voluntaria y un reclutamiento mayor en las fibras de contracción de tipo I (ST) y II (FT mix), que son las primeras en activar sus unidades motoras para realizar el movimiento (Hohmann et al. 2005; Weineck, 2005; González et al. 2011; Keijo et al. 2007; Álvarez et al., 2006). En este trabajo, no se define el tipo de fibra reclutado por el programa de entrenamiento, pero los resultados arrojados de la actividad neural, frente a un mismo estímulo, aplicado en dos momentos diferentes, después de un programa de quince semanas de entrenamiento de la fuerza, utilizando el método progresivo escalonado, nos muestra una disminución en la necesidad de reclutamiento de unidades motoras y su potencia de inervación, es decir, disminuye el trabajo muscular en cuanto a la necesidad de fuerza. Con relación a la resistencia, según la media de la frecuencia de inervación (MDF), el estudio indica que existe una disminución en la mayoría de los casos por adaptación fisiológica y no como lo refieren Algunos estudios, que probaron que la fatiga acumulada y la producción de ácido láctico disminuyen el potencial de acción de la unidad motora, razón por la que disminuye la frecuencia de inervación e interacción con la célula muscular (González et al. 2011).

Los protocolos utilizados en este trabajo investigativo, no permiten que el deportista alcance niveles máximos de esfuerzo, tampoco realiza esfuerzos prolongados, por lo que no alcanza niveles altos de fatiga la cual puede influir en la respuesta de la célula muscular al estimulo (González et al. 2011; Keijo et al. 2007; Alvarez et al., 2006). La electromiografía (EMG) resulta ser el un método diagnostico útil para validar hipótesis, y simplificarlas en el desarrollo de modelos dinámicos del aparato locomotor. Sin embargo, hoy existe confusión respecto a la EMG debido a la deficiente compresión de los principios físicos que rigen esta metodología (la naturaleza de la señal 

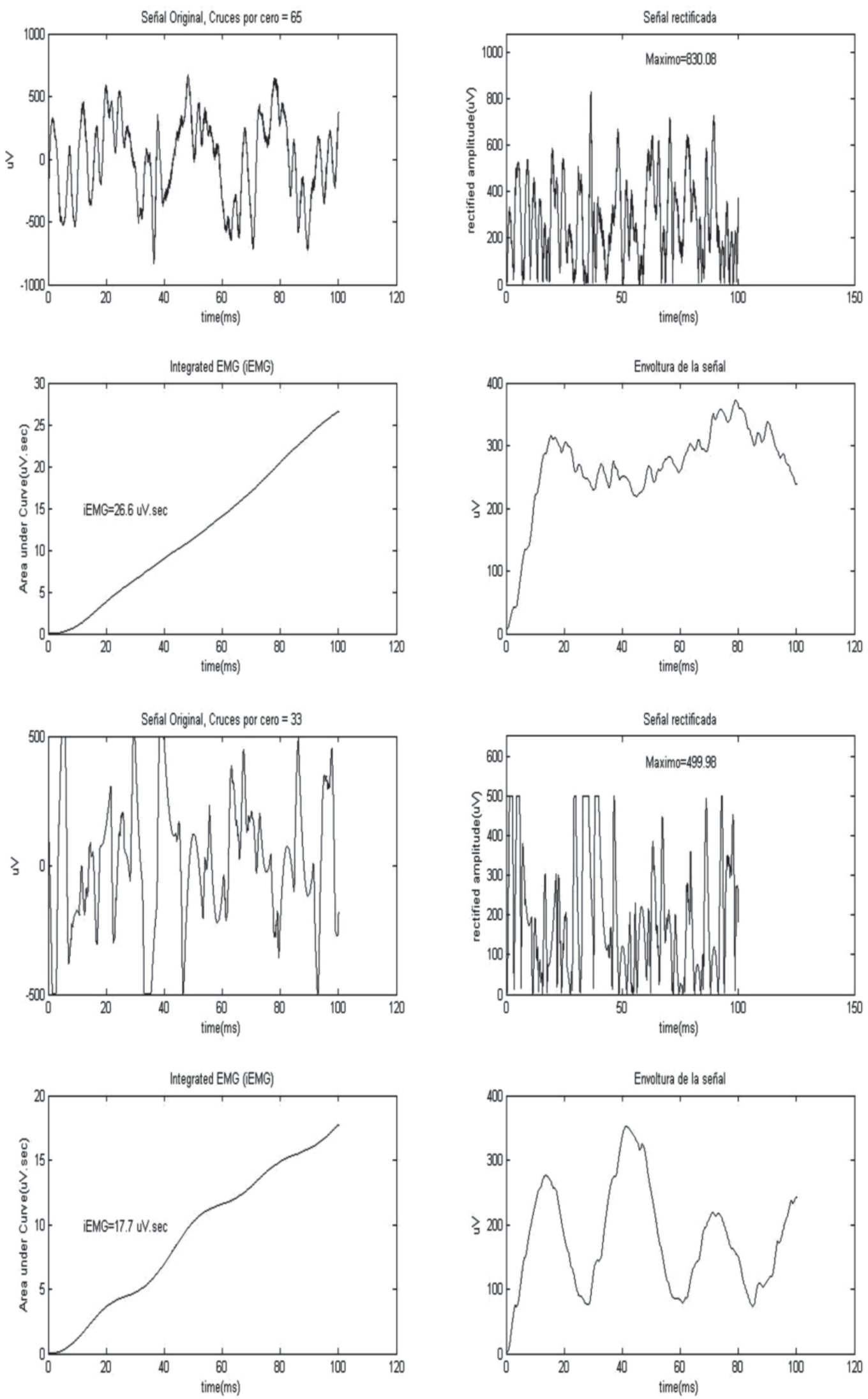

Figura 3. EMG Pretest-Postest trapecio izquierdo deportista 3. 
registrada y el procesamiento de los datos). El objetivo de este trabajo ha sido describir los cambios que ocurren a nivel muscular después de un programa de fuerza, los fundamentos de la EMG son base de sustento para la descripción de los datos. Conviene aclarar que la señal electromiográfica registrada no depende solo de la intensidad de la acción muscular, sino también de la calidad de la instrumentación utilizada y el tratamiento adecuado del electromiógrafo, porque permite conocer las implicaciones objetivas del entrenamiento específico en el estudio de la técnica deportiva, en las diferentes modalidades de los deportes de precisión.

El análisis de los resultados demostró que la fuerza juega un papel decisivo en la ejecución técnica. En muchos casos (no en todos), el fallo técnico no se produce por falta de coordinación o habilidad, sino por la deficiencia de fuerza en los grupos musculares que intervienen en una fase concreta del movimiento (Billat, 2002). Con el entrenamiento direccionado al desarrollo de la fuerza en los arqueros de selección Colombia, surgieron adaptaciones concretas, relacionadas con el plan de trabajo, la individualización y cumplimiento cabal del mismo, mejorando la respuesta al estímulo referido (el libraje del arco) evidenciado en la disminución de la fuerza para la respuesta motora haciéndola más fina.

Se evidenció una disminución en la activación de las unidades motoras, la reducción del PUM y, por ende, la menor necesidad de desarrollo de la fuerza y un cambio morfológico de los deportistas. Después del proceso de entrenamiento específico, en relación con los objetivos biológicos y pedagógicos inherentes a la planificación, se evidenciaron cambios en la masa magra, el peso y en los perímetros del tren superior (todos los valores aumentaron). De la misma forma, se produjo una hipertrofia tendinosa, sin abombamientos degenerativos en los casos que cumplieron por encima del $80 \%$ de lo planificado, en los músculos evaluados e implicados en el disparo.

Conflicto de intereses: Los autores del presente artículo, manifestamos que este fue preparado y revisado con la participación de todos los autores, quienes declaramos que NO existe ningún conflicto de interés que ponga en riesgo la validez de los resultados presentados.

Financiación: El proceso investigativo fue financiado por la Universidad Autónoma de Manizales dentro del proceso académico de la maestría en Intervención Integral en el deportista y además contó con la participación de la Federación Colombiana de tiro con arco y del comité Olímpico Colombiano».

\section{Referencias bibliográficas}

Álvarez, R.; Santos, C.; Medina, H. 2006. Desarrollo histórico y fundamento teóricos de la electromiografía como medio diagnóstico. Rev. Cubana Med. Milit; 35(4):-0-0.

Berdejo, R.; González, J.M. Strength training in young tennis players. J. Sport and Health Res. 1(1)(2006):46-55.

Billat, V. Entrenamiento deportivo: metodología del entrenamiento deportivo. Fisiología y metodología del entrenamiento de la teoría a la práctica. $1^{\mathrm{a}}$ ed. Barcelona: Editorial Paidotribo, 2002, p. 130-134.

Carratala, V.; Carques L. Valoración de la fuerza explosiva, elástico explosiva de los judokas infantiles y cadetes del equipo nacional español, 2003.

Departamento de Educación Física y Deportiva. Universidad de Valencia. España, p. 1-6.

Ergen, E.; Hibner, K. Sports medicine and science in archery. Muscular Activation strategies in archery. In: Ergen, E.; Hibner, K. (eds). 2a ed. Ankara: FITA; p. 22-26, 2008.

Ergen, E.; Hibner, K. Sports medicine and sciece in archery Shoulder Strengtening exercises for injury prevention in archery. In: Ergen, E.; Hibner K. (eds). $1^{\mathrm{a}}$ ed. Ankara: FITA; p. 87- 89, 2004.

Gonzales, B.J.; Gosrostiaga, E. Fundamentos del entrenamiento de la fuerza aplicación al alto rendimiento, comité olímpico español y Universidad Autónoma de Madrid. p. 45-60, 2011.

Hohmann, A.; Letzelter, M.; Lames, M. Capacidad de rendimiento en el deporte. Fuerza en el deporte. Introducción a la ciencia del entrenamiento. $1^{\mathrm{a}}$ ed. Editorial Paidotribo; $\mathrm{p}$. 81-104, 2005.

COLDEPORTES. Instituto Colombiano de la Recreación y el Deporte. del deporte: definiciones y clasificaciones. In: Kinesis, editor. Legislación deportiva, ley 181 de 1995. $1^{\text {a }}$ ed. Bogotá: Coldeportes; p. 21-22, 2001. 
Herrera, A. H. Contraste pre isométrico como método para el incremento de la fuerza específica en atletas de tiro con arco. Disponible desde Internet en: www.simedar. org/archivos/investigacion/.../metodo_tiroconarco.pdf (con acceso 12 03-2012), 2009.

Lee, K.; Benner, T. Total Archery. Inside the archery. Posture. $1^{\text {a }}$ ed.: Astra LLC, California USA; p. 16-19, 2009.

Lee, K.; Bont, R. Total Archery. Physical Training. 1 ed. Republic of Corea: SAMICKSPORTSCO. p. 14-31-44, 2005.

Lee, K.; Bont R. Total Archery. Biomechanics. In: editions, editor. 1 ed. Republic of Corea: SAMICK SPORTS CO; p. 75-78, 2005.

Lee, K.; Benner, T. Total Archery. Shoulder Alignament. In: Adamson Spencer, editor. 1 ed. California u.s: Astra LLC; p. 190-196, 2009.

Lee, K. Total Archery. Technical performance analysis. 1 ed. Republic of Corea: SAMICK SPORTS CO; pp. 91, 92-97, 2005.

Hakkinen, K.; Newton, R.U.; Evans, W.J.; Campbell, W.W.; Gordon, S.E.; Gotshalk, L.A.; Mccormick, M.; Volek, J.S.; Nindl, B.C.; Hakkinen, A. Cambios en la Morfología Muscular, Actividad Electromiográfica, y en las Características de Producción de Fuerza durante el Entrenamiento Progresivo de Sobrecarga, en Hombres Jóvenes y Ancianos. G-SE Standard. 03/08/2007. g-se.com/a/784/. Disponible desde Internet en: http://www.g-se.com/a/784/ cambios-en-la-morfologia-muscular-actividad-electro miografica-y-en-las-caracteristicas- de-produccion-defuerza-durante-el-entrenamiento-progresivo-desobrecarga-en-hombres-jovenes-y-ancianos/ (con acceso 15-03-2012), 2007.

Keijo, H.; Robert, N. Cambios en la Morfología Muscular, Actividad Electromiográfica, y en las Características de Producción de Fuerza durante el Entrenamiento Progresivo de Sobrecarga, en Hombres Jóvenes y Ancianos. Centro de Investigación Neuromuscular y Departamento de Biología de la Actividad Física, Universidad de Jyvaskyla, Finlandia, 2007.

Mansilla, M.; Villa J.; García J.; López C. Comparación de diferentes manifestaciones de fuerza y flexibilidad entre luchadores de lucha leonesa y judokas. I Congreso de la Asociación Española de Ciencias del Deporte. Universidad de Extremadura; Cáceres. marzo. Disponible desde Internet en: www.unex.es/eweb/cienciadeporte/congreso/ 00\%20cac/RD/ED/11fuerza.pdf (con acceso....., 2000.

Torres, A.; Cardona, J.; Stephens, R. Efectos del entrenamiento pliométrico en miembros superiores en la fuerza aplicada y la precisión de tiro con bola, en jóvenes beisbolistas de 12 a 14 años. Inder Envigado. Udea, p. 102-116, 2005.

Weineck J. Entrenamiento de la fuerza. Entrenamiento Total. 1 ed. Editorial Paidotribo; pp. 215-240. Wise L. Muscle activity during Back tension. In: Series of out door sports, editor. Core archery. 16 ed. United States: On target; 2007. p. 90-92, 2005. 